\title{
Spectral Analysis on Sub-tidal Variability of Salinity in Modaomen Waterway of Pearl River Estuary, China
}

\author{
Yuan Ying Chen ${ }^{1,2, a}$, Xiao Ling Yin ${ }^{1, b^{*}}$, Dong Lin Bai ${ }^{1, c}$ and Li Cheng Li ${ }^{1, d}$ \\ ${ }^{1}$ School of civil engineering and transportation, SCUT, Guangzhou, China \\ ${ }^{2}$ Lab. Environmental Mechanics, Inst. Mechanics, CAS, Beijing, China \\ achenyuanying11@mails.gucas.ac.cn, ${ }^{\mathrm{b}}$ arxlyin@scut.edu.cn, \\ cbaidonglin001@gmail.com, ${ }^{d}$ lilicheng123@gmail.com
}

Keywords: Estuary, Sub-tidal, Salinity, Spectral analysis, Pearl River

\begin{abstract}
Salinity and tidal range time series observed in Modaomen waterway was analyzed in power spectrum method, and both showed the period of half month (14.22d). Moreover, the salinity and tidal range time series were coherent at that period through cross spectrum analysis. Besides, the phase analysis at the period of $14.22 \mathrm{~d}$ showed that, within the estuary, the salinity time series upstream lagged that of the downstream, and the response time of salinity time series to the tidal range time series was about 9-10d, increasing upstream. But the phase of salinity time series and response time to the tidal range at the estuary mouth did not correspond with the laws within the estuary. The response time of salinity to the tidal range was about $12 \mathrm{~d}$ there.
\end{abstract}

\section{Introduction}

Modaomen is one of the eight mouths of the Pearl Delta, and its upstream is the main source of water for some important cities, such as Zhongshan, Zhuhai and Macau (Fig.1). However, in the recent years, the development of the area, both in agriculture and industry, is restricted because of the salt water intrusion.

The diurnal and semi-diurnal salinity in Modaomen waterway was not influenced by the fresh water discharge when the discharge changed little, but correlated with tide instead[1,2]. The diurnal change of salinity was decided by the diurnal tide, which was about $5 \mathrm{~h}$ ahead of salinity time series[1,3]. And at the period of half month, salinity corresponded well with tide, but they were not synchronous in time[1,3]. The maximum salinity appeared 3-5d ahead of the maximum tidal range[4].
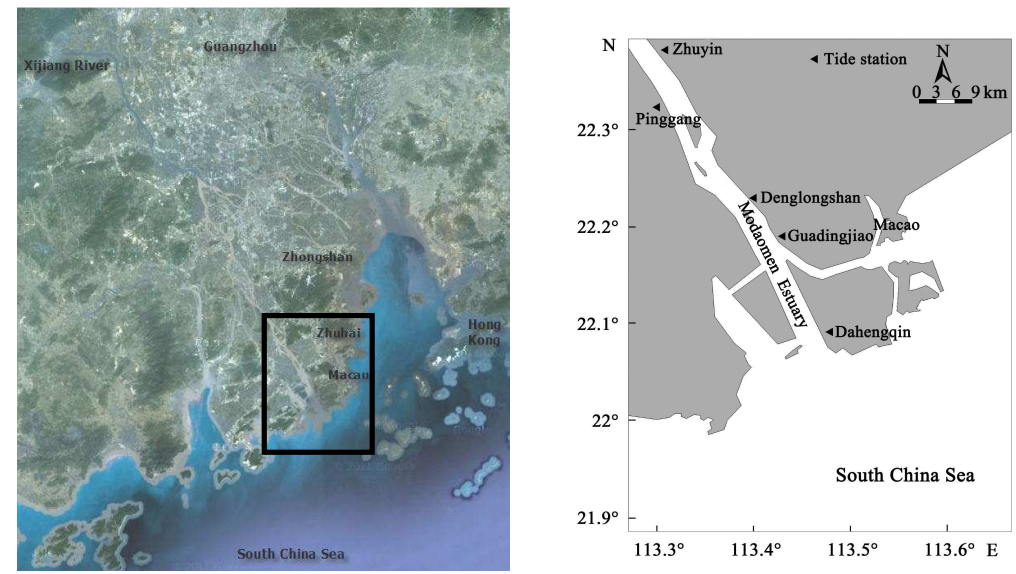

Fig.1 Location map of Modaomen Mouth and the locations of observing stations

Spectrum analysis, useful in observing dominant frequency and analyzing coherence, is frequently used in identifying the periods of tidal level and its influential factor[5,6,7]. It has also been applied in studying the correlation of the discharge and salinity in the Changjiang Estuary, with the result that salinity at low frequency was primarily affected by discharge[8]. 
In this paper, the observed data of salinity, tidal level and tidal range around the mouth of Modaomen waterway (Fig. 1) in dry season were analyzed through time series analysis method. And we mainly focused on the sub-tidal fluctuations, intending to find out more about the laws of water intrusion in Modaomen.

Data discussed in this paper were observed tidal level and surface salinity from Oct 1st,2007 to Feb 29th ,2008, and the surface salinity was taken every half hour and tidal level every five minutes. Factors such as fresh water discharge and wind were not discussed here.

\section{The Power Density Distribution and Correlation Between Salinity and Tide}

In this section, data from Denglongshan station were discussed as representative. Through power spectral analysis we got the power density distribution, and through cross spectral analysis we got the coherence between salinity and tidal range.

The Power Spectral Analysis of Salinity. As shown in Fig.2, the power density increased towards lower frequency, and there was a spectral peak at $14.22 \mathrm{~d}$, and two secondary peaks at $1.07 \mathrm{~d}$ and $0.52 \mathrm{~d}$, which corresponded to the semi-monthly, diurnal and semi-diurnal salinity periods, respectively. Semi-monthly salinity contributed $25.97 \%$ of the total spectral energy; diurnal and semi-diurnal bands accounted for $4.01 \%$ and $1.39 \%$, respectively. Thus, it could be indicated that the salinity were predominantly semi-monthly, and studying the period of $14.22 \mathrm{~d}$ was paramount for salinity prediction and driving mechanism analysis.

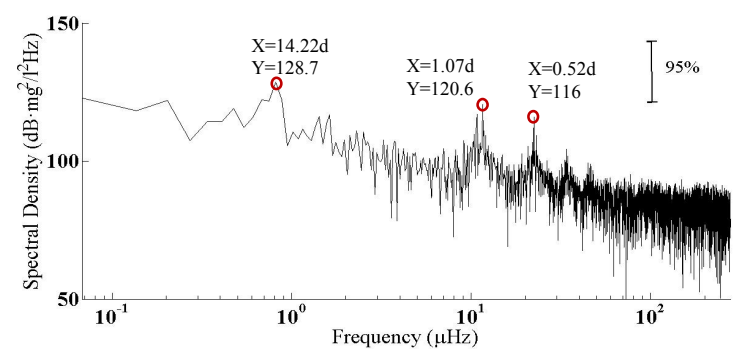

Fig. 2 Power spectrum analysis of salinity

The Power Spectrum Analysis of Tide. As the result of tidal level power spectral analysis showed (Fig.3), the power density increased towards lower frequency. The peaks of the spectrum were at $1.00 \mathrm{~d}$ and $0.52 \mathrm{~d}$, respectively, and the amplitudes at these two frequencies were approximately equal. There was no peak at the frequency corresponding to semi-monthly period.

And the spectral analysis of the tidal range(Fig.4) showed a peak at 14.22d, which contributed $43.42 \%$ of the total energy .

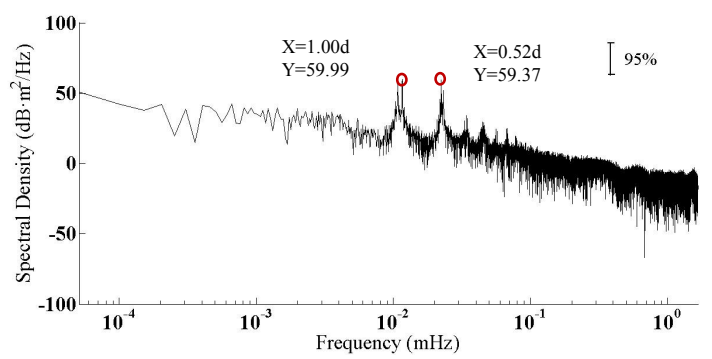

Fig. 3 Power spectrum analysis of tidal level

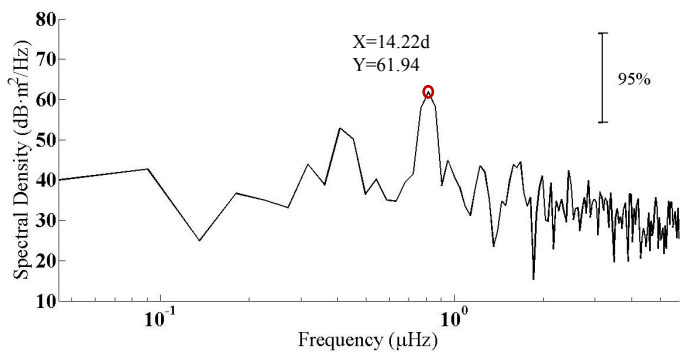

Fig. 4 Power spectrum analysis of tidal range

The Cross Spectrum of Salinity and Tidal Range. According to the spectrum analysis above, both of the salinity and tidal range time series of Denglongshan station showed the period of 14.22d, at which the salinity and tidal range were probably coherent. Further, the salinity and tidal range time series of Denglongshan station were analyzed using cross spectrum, and the result showed that the coherence coefficient between salinity and tidal range at the period of $14.22 \mathrm{~d}$ reached 0.877 , far exceeding 95\% confidence level of 0.226 ( Fig.5 ). 
To examine the generality of the result above, salinity and tidal range data from Dahengqin station at the mouth of Modaomen was calculated. As shown in Fig 6, the coherence coefficient between salinity and tidal range at the period of $14.22 \mathrm{~d}$ reached 0.567 , greater than $95 \%$ confidence level of 0.291 . The cross spectrum analysis proved that tidal range was an important factor of salinity at the period of half month.

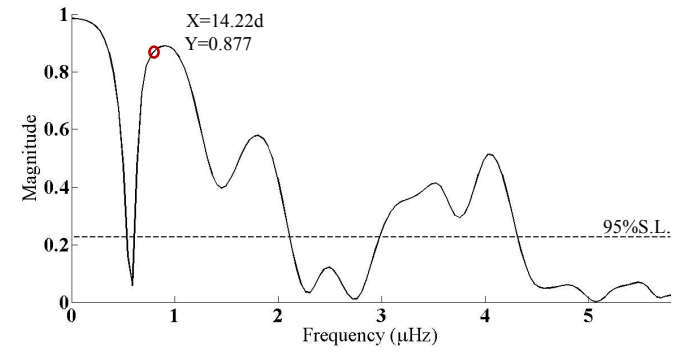

Fig. 5 Cross spectrum analysis of salinity and tidal level at Denglongshan station

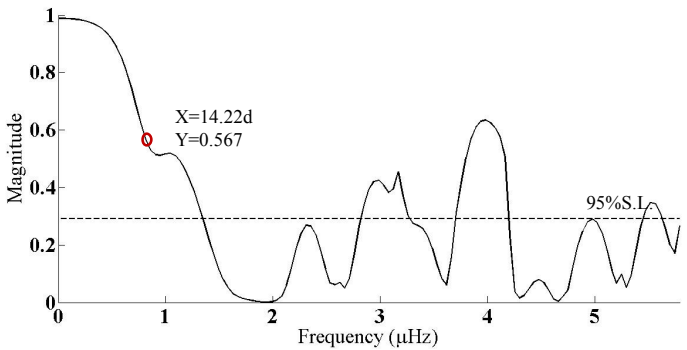

Fig. 6 Cross spectrum analysis of salinity and tidal level at Dahengqin station

\section{The Phases of Salinity and Tidal Range Time Series at the Period of Half Month}

The Phase Correlation of Salinity from Different Stations. Phases of time series at different frequency were calculated using a fast Fourier transform(FFT) algorithm. Table 1 showed salinity time series phases at the frequency corresponding to the $14.22 \mathrm{~d}$ period of Zhuyin, Pinggang, Denglongshan, Guadingjiao and Dahengqin stations(Fig.1), respectively. Salinity phases of stations within the estuary decreased upstream, which indicated the salinity time series downstream leaded that of the upstream. However, the salinity of Dahengqin station, lying at the mouth of Modaomen, lagged behind those within the estuary. And the salinity phase of Dahengqin station was $55.82^{\circ}$ behind Guadingjiao, representing a time lag of $2.2 \mathrm{~d}$ of salinity time series. To examine the generality of the time lag of Dahengqin station, phases of salinity time series in dry season of 06-07, 08-09 was calculated. As shown in Table 2, the salinity time series at Dahengqin lagged behind Guadingjiao for all the three years, which indicated that the phenomenon was common; but the explanation of the phenomenon needs further investigations and discussions.

Table 1 Salinity phases of different stations at the frequency corresponding to $14.22 \mathrm{~d}$ period $\left({ }^{\circ}\right)$

\begin{tabular}{cccccc}
\hline Station & Zhuyin & Pinggang & Denglongshan & Guadingjiao & Dahengqin \\
\hline Phase & -64.38 & -62.31 & -54.06 & -43.63 & -99.45 \\
\hline
\end{tabular}

Table 2 Salinity phases of Dahengqin and Guadingjiao at the frequency corresponding to $14.22 \mathrm{~d}$ period $\left({ }^{\circ}\right)$

\begin{tabular}{cccc}
\hline Year & Guadingjiao & Dahengqin & Phase difference \\
\hline $07-08$ & -43.63 & -99.45 & 55.82 \\
$08-09$ & -11.37 & -47.62 & 36.25 \\
$09-10$ & -107.39 & -142.02 & 34.63 \\
\hline
\end{tabular}

Table 3 The response time of salinity to tidal range at different stations

\begin{tabular}{ccccc}
\hline Station & Zhuyin & Denglongshan & Guadingjiao & Dahengqin \\
\hline Phase difference $\left({ }^{\circ}\right)$ & 257.20 & 243.87 & 231.80 & 306.13 \\
Response time $(\mathrm{d})$ & 10.16 & 9.63 & 9.16 & 12.09 \\
\hline
\end{tabular}

The Salinity Response to Tidal Range. Phases of salinity and tidal range time series at the frequency corresponding to $14.22 \mathrm{~d}$ period at the stations of Zhuyin, Denglongshan, Guadingjiao and Dahengqin were calculated here, and converted into response time in day. As shown in Table 3, the 
salinity time series lagged behind tidal range time series. And the response time of salinity to tidal range, which increased upstream, was about 9-10d at the stations within the estuary, while the response time at the Dahengqin station, lying at the mouth of the estuary, was $12.09 \mathrm{~d}$. Thus, according to tidal data and the response time, the variability of salinity at the period of half month could be predicted.

From the analysis above, it could be indicated that the characteristic of salinity time series and response time to the tidal range at the estuary mouth were different from those within the estuary. But detailed explanation of the phenomenon needs further analysis and discussions

\section{Conclusions}

According to the spectrum analysis, both of the salinity and tidal range time series showed the period of $14.22 \mathrm{~d}$, and the salinity and tidal range time series were coherent at that period. The cross spectrum analysis proved that tidal range was an important factor of salinity variability at the period of half month.

The phase analysis showed that, within the estuary, the salinity time series upstream lagged that of the downstream, and the response time of salinity time series to the tidal range time series was about 9-10d. But the characteristic of salinity time series and response time to the tidal range at the estuary mouth did not correspond with the laws within the estuary. The response time of salinity to the tidal range was about $12 \mathrm{~d}$ there.

In this paper, spectral analysis was used, and the result was not only accordant with the past research but also promoted new understanding of the problem. Thus, we can say that the application of spectral analysis method in salt water intrusion was effective and it could be considered as a supplementary method of research in salt water intrusion.

\section{Acknowledgements}

The work was sponsored by NSFC for General Program Project (10972080). The authors acknowledge the helpful discussions and data collections provided by the colleagues from the Ocean Center of Sun Yat-Sen University.

\section{References}

[1] Yin Xiaoling: Study on Scheduling of Main Reservior on Pearl River for Estuarine Fresh Water Providing Based on Digital Basin Model. PhD thesis. Tsinghua University, China, 2008[In Chinese]. [2] Liu Jiebin, Bao Yun and Huang Yuming: Chinese Journal of Theoretical and Applied Mechanics, Vol.42,No.6(Nov,2010), p.1098[In Chinese].

[3] Liu Jiebin and Bao Yun: Acta Scientiarum Naturalium Universitatis Sunyatseni, Vol.47,Sup.2 (Nov,2008), p.122[In Chinese].

[4] Zhu Sanhua, Shen Hangun and Lin Huanxin and Suo Xiaobo: Pearl River Modern Construction, Vol.6(Dec,2007),p.1[In Chinese].

[5] Belmiro M.Castro and Thomas N.Lee: Journal of Geophysical Research, Vol.100, NO.C8, p.16,045, 1995.

[6] Joanne O'Callaghan, Charitha Pattiaratchi and David Hamilton : Continental Shelf Research, 27 (2007),p.1947.

[7] Zhao Baoren and Cao Deming: Oceanologia Et Limnologia Sinica, Vo1.18, No.6(Nov,1987),p. 563[In Chinese].

[8] Shen Huanting, Wang Xiaochun and Yang Qingshu: Acta Oceanologica Sinica, Vo1.22. No.4(July,2000),p.17[In Chinese]. 
Advances in Environmental Science and Engineering

10.4028/www.scientific.net/AMR.518-523

Spectral Analysis on Sub-Tidal Variability of Salinity in Modaomen Waterway of Pearl River Estuary, China

10.4028/www.scientific.net/AMR.518-523.1982 